\title{
An Integrated Risk Management Method: VaR Approach*
}

\author{
Hailiang Yang \\ The University of Hong Kong, Hong Kong
}

This article presents a simple methodology for computing Value at Risk $(\mathrm{VaR})$ for a portfolio of financial instruments that is sensitive to market risk, rating change, and default risk. An integrated model for market and credit risks is developed. The Jarrow, Lando and Turnbull model (the Markov chain model) is used to represent the dynamics of the credit rating. Procedures for calculating VaR are presented. Numerical illustration results are included (JEL G10, D81).

Keywords: credit rating, default risk, integrated risk management, Markov chain, value at risk.

\section{Introduction}

Broadly speaking, risk management includes the management of both financial and non-financial risk. Financial risk includes market risk and credit risk whereas non-financial risk includes operational risk, regulatory risk, legal risk, and event risk. In this paper, we shall be focusing on financial risk.

Alfred Steinherr (1988) stressed the significance of modern risk management by calling it "one of the most important innovations of the 20th Century."

Investment banks and financial institutions around the world seek various methods and tools to manage the risks they face. Due to the

*The author would like to thank the editor-in-charge, Professor Yin-Wong Cheung, and the referee for many helpful comments, suggestions, and corrections concerning previous versions of this paper. This work was supported by a grant from the Research Grants Council of the HHSAR (Project No: HKU 7168/98H).

(Multinational Finance Journal, 2000, vol. 4, no. 3\&4, pp. 201-219)

(C) Multinational Finance Society, a nonprofit corporation. All rights reserved.

DOI: $10.17578 / 4-3 / 4-4$ 
recent rapid development of the derivative market, the tasks involved in risk management have become even more challenging than before. This has accelerated the development of more advanced techniques of risk management. Traditionally, volatility was the risk measure commonly used by the finance community, but recently Value at Risk (VaR) has also become a very popular measure of risk. $\mathrm{VaR}$ is an attempt to summarize the total risk of a portfolio with a single number that is the statistical estimation of a portfolio loss. The VaR is the amount, with a given (small) probability, that we may lose over a given (typically short) holding period. Along with this probability there is also a possibility that we may lose more. Both Embrechts (2000) and J. P. Morgan's "Risk Metrics $^{\mathrm{TM}}$ - Technical Document" give an introduction to VaR while a useful survey is presented by Duffie and Pan (1997). Credit risk has perhaps become the key risk management challenge since the late 1990s. J. P. Morgan's "Introduction to CreditMetrics" provides an overview of this subject. Ong (1999) provides a coherent framework for thinking about and modeling bank credit risk. In 1997, Jarrow, Lando and Turnbull (JLT) proposed a Markov chain model for valuing risky debts that explicitly incorporate a firm's credit rating as an indicator of the likelihood of default. Kijima and Komoribayushi (1998) studied this model further. More recently, Arvanitis, Gregory and Laurent (1999) have built models for credit spreads and used a Markov chain to represent the credit rating dynamics.

Of course, in most markets nowadays, both market and credit risks have been measured and quantified using agreed methodologies. However, risk managers are increasingly seeking quantified and integrated risk management tools that can capture market exposure, rating change, and default risk. Recent developments in integrating market risk and credit risk analysis were presented by Huang (2000). This paper presents a simple model for integrated risk management. We assume that firms face both market and credit risks. The credit risk ratings are modelled by using the JLT (1997) Markov chain model (see also Kijima and Komoribayashi (1998)). It is well known that VaR has been used successfully in measuring and managing market risk. Due to its skewed distribution, modelling credit risk is neither analytically nor practically easy. In the first part of this paper, we present an integrated risk assessment within a VaR context and discuss VaR calculation procedures. The analytical tools in this paper are similar to those in Yang 
(2000). In Yang (2000), an insurance risk model with credit risk was considered. Some commonly discussed problems in risk theory, such as ruin probability, ruin time distribution, severity of ruin, and joint distribution of surplus before and after ruin, were addressed. In the second part of this paper, we use some of the results from Yang (2000) in the context of credit risk rather than ruin theory. Some numerical results illustrating how to analyze default risk are included.

This paper proceeds as follows. Section II presents the problem formulation and the procedures used for VaR calculation. An illustrative example is also included. Section III discusses the distributions of the default probabilities and the time of default. Some illustrative numerical results are included as well. The final section contains some concluding remarks.

\section{An Integrated Method for Managing Market and Credit Risks}

In this paper, we consider a firm that could be either a financial corporation or an insurance company. At the beginning of each time interval, a rating agency will provide a credit rating to assess the firm's abilities to meet its debt obligations (to pay possible claims in an insurance company case). The rating of the firm is based on its financial situation and initial surplus. The firm also faces exposure to marketbased financial risk. We will propose an integrated method to measure the risk faced by the firm.

\section{A. Credit and Market Risk Models}

This paper uses a Markov chain model for the dynamics of the firm's credit ratings (see JLT (1997)). The only difference here to JLT (1997) is that the default state is not included in the state space of the Markov chain.

Let $I_{t}$ be a time-homogeneous Markov chain with state space $N=\{1$, $2, \ldots, k\}$ where state 1 represents the highest credit class and state $k$ represents the lowest. According to Moody's ratings, state 1 can be thought of as $A a a$ and state $k$ as $C a a$, whereas according to $S \& P$ 's ratings, state 1 can be thought of as $A A A$ and state $k$ as $C C C$. In our formulation, we are not including the default state in the transition 
matrix. Let

$$
q_{i j}=P\left\{I_{t+1}=j \mid I_{t}=i\right\}, \quad i, j \in N, \quad t=0,1,2, \ldots
$$

be the one-step transition probabilities. The transition matrix of the Markov chain $I_{t}$ can then be written as

$$
Q=\left[\begin{array}{ccccc}
q_{11} & q_{12} & \cdots & q_{1 k-1} & q_{1 k} \\
q_{21} & q_{22} & \cdots & q_{2 k-1} & q_{2 k} \\
\vdots & \vdots & & \vdots & \vdots \\
q_{k-11} & q_{k-12} & \cdots & q_{k-1 k-1} & q_{k-1 k} \\
q_{k 1} & q_{k 2} & \cdots & q_{k k-1} & q_{k k}
\end{array}\right]
$$

Let $u$ be the initial surplus of the firm and let $\Delta X_{n}^{i}$ be the portfolio change in the $n^{\text {th }}$ time interval when the firm's credit rating in the time interval $n$ is of class $i$. The surplus of the firm at time $n$ can then be written as

$$
U_{n}=u+\sum_{m=1}^{n} \Delta X_{m}^{I_{m-1}}=u+\Delta Y_{n}
$$

where we assume that $\Delta X_{m}^{i}, i=1, \ldots, k, m=1,2, \ldots$ are independent random variables. If $U_{n} \leq 0$ we say that default occurred at time $n$.

We will assume that for any fixed $i=1, \ldots, k, \Delta X_{m}^{i}, m=1,2, \ldots$, are identically distributed and $\Delta X^{1}, \ldots, \Delta X^{k}$ are independent but may have different distributions. For example, we may assume that $\Delta X^{1}$ only takes positive values, that $\Delta X^{k}$ may take negative values with high probabilities, and that the process will stop if default occurs. The market risk focuses on the firm's investments in the market. This paper models the market risk by assuming that the portfolio's change in each time interval is random. For example, if we assume that the investment return follows a normal distribution, we can also assume that the portfolio change in each interval follows a normal distribution. Although the firm investments in the market do not depend on the credit rating, the firm's portfolio change in each time interval depends on both the firm's 
investments in the market and the rating of the credit. Therefore, in our model, the market risk is built in the distribution of $\Delta X_{m}^{i}$. From now on, the distribution of $\Delta X^{i}$ is denoted by $F^{i}(x)$.

\section{Remarks:}

1. The assumption that $I_{t}$ is a time-homogeneous Markov chain is only made to simplify notations. All the analyses in this paper work, even if $I_{t}$ is a non-homogeneous Markov chain.

2. The probability transition matrix, $Q$, can be estimated using historical data. We may also be able to use some results currently available in the literature. For example, from J. P. Morgan's home page (http://www.riskmetrics.com).

3. The assumption that for any fixed $i \in\{1, \ldots, k\}, \Delta X_{n}^{i}, n=1,2, \ldots$, is i.i.d. can be relaxed. If we now assume that the business will expand and that the growth rate over each time interval is a constant, the following model can be used for the dynamics of the surplus process:

$$
U_{n}=u+\sum_{m=1}^{n} \Delta X_{m}^{I_{m-1}}(1+r)^{m-1}
$$

where $r$ is the business growth rate. With all other assumptions remaining the same as before, the results presented in this paper can be extended to this model without difficulty. Mathematically, model(4) is the same as that in Yang (1999) where an insurance risk model with interest incomes was considered.

\section{B. VaR approach}

Suppose we are at time 0 and there is a $5 \%$ probability that we may incur a certain amount of loss or more over the next time period, say of one day. This amount of portfolio loss is what we are concerned about. We call it the $95 \% \mathrm{VaR}$. It can also be interpreted as a minimum margin requirement. The one-day $\mathrm{VaR}$ at a $5 \%$ confidence level can be defined using the following equation (the confidence percentage can be changed to any other value): 


$$
\operatorname{VaR}_{5 \%}\left(\Delta Y_{1}\right)=-\inf \left\{y \in \mathbb{R} \mid F_{\Delta Y_{1}}^{i_{0}, u}(y)>5 \%\right\},
$$

where $F_{\Delta Y_{1}}^{i_{0}, u}$ is the conditional probability distribution of $\Delta Y_{1}$ knowing that $I_{\mathrm{o}}=i_{0}$ and $U_{0}=u$. Let $T=\inf \left\{n ; U_{n} \leq 0\right\}$. The stopping time, $T$, is called the default time. In practice, we may also become interested in the VaR for more than one day, say for $n$ days. The $n$-day VaR can be defined in a similar way to the one-day VaR. However, since the default may occur in less than $n$ days, we need to divide the problem into two cases. This motivates us to consider the following problems: For $y$ $\leq u$,

$$
P\left\{\Delta Y_{j} \leq-y, \exists j \leq n, T>n \mid I_{0}=i_{0}, U_{0}=u\right\}=5 \% .
$$

For $y \geq u$,

$$
P\left\{\Delta Y_{T} \leq-y, T \leq n \mid I_{0}=i_{0}, U_{0}=u\right\}=5 \% .
$$

The probability on the left hand side of (5) can be calculated by the following recursive method:

$$
\begin{gathered}
P\left\{\Delta Y_{j} \leq-y, \exists j \leq n, T>n \mid I_{0}=i_{0}, U_{0}=u\right\} \\
=\sum_{j=1}^{n} h_{n, j}^{i_{0}}(u, y),
\end{gathered}
$$

where

$$
\begin{aligned}
& h_{n, j}^{i_{0}}(u, y)=P\left\{\Delta X_{1}^{i_{0}}>-y, \ldots,-u<\Delta X_{1}^{i_{0}}+\cdots\right. \\
& \left.+\Delta X_{1}^{I_{j-1}} \leq-y, \ldots, \Delta X_{1}^{i_{0}}+\cdots+\Delta X_{n}^{I_{n-1}}>-u\right\},
\end{aligned}
$$

and $h_{n, j}^{i_{0}}(u, y)$ can be calculated using the following procedure: For $j$ $=0$ and $n \geq 2$,

$$
h_{1,0}^{i_{0}}=1-F^{i_{0}}(-u)
$$




$$
h_{n, 0}^{i_{0}}(u)=\sum_{i=1}^{k} q_{i_{0} i} \int_{-u}^{\infty} h_{n-1,0}^{i}(u+x) d F^{i_{0}}(x)
$$

For $j=1$ and $n \geq 2$,

$$
\begin{gathered}
h_{1,1}^{i_{0}}(u, y)=P\left\{-u<\Delta X_{1}^{i_{0}} \leq-y\right\}=F^{i_{0}}(-y)-F^{i_{0}}(-u), \\
h_{n, 1}^{i_{0}}(u, y)=\sum_{i=1}^{k} q_{i_{0} i} \int_{-u}^{-y} h_{n-1,0}^{i}(u+x) d F^{i_{0}}(x) .
\end{gathered}
$$

After obtaining $h_{m, 1}^{i}(u, y)$ for $m \leq n$ we consider the case $j \geq 2$ :

$$
h_{n, j}^{i_{0}}(u, y)=\sum_{i=1}^{k} q_{i_{0}} \int_{-y}^{\infty} h_{n-1, j-1}^{i}(u+x, y+x) d F^{i_{0}}(x) .
$$

The probability on the left-hand side of equation 6 can be calculated using the following recursive equation:

$$
\begin{aligned}
P\left\{\Delta Y_{T} \leq-y, T\right. & \left.\leq n \mid I_{0}=i_{0}, U_{0}=u\right\}=H_{n}^{i_{0}}(u, y) \\
& =\sum_{m=1}^{n} h_{m}^{i_{0}}(u, y),
\end{aligned}
$$

where

$$
\begin{gathered}
h_{m}^{i_{0}}(u, y)=P\left\{\Delta X_{1}^{i_{0}}>-u, \ldots, \Delta X_{1}^{i_{0}}+\cdots+\Delta X_{m-1}^{I_{m-2}}>-u,\right. \\
\left.\Delta X_{1}^{i_{0}}+\cdots+\Delta X_{m}^{I_{m-1}} \leq-y\right\},
\end{gathered}
$$

and $h_{m}^{i_{0}}(u, y)$ can be calculated recursively by:

$$
h_{1}^{i_{0}}(u, y)=P\left\{\Delta X_{1}^{i_{0}} \leq-y\right\}=F^{i_{0}}(-y),
$$


Remarks:

$$
\begin{gathered}
h_{m}^{i_{0}}(u, y)=\sum_{i=1}^{k} q_{i_{0} i} \int_{-u-}^{\infty} h_{m-1}^{i}(u+x, y+x) d F^{i_{0}}(x), \\
m=2,3, \ldots
\end{gathered}
$$

1. If equation 6 has a solution, there is $5 \%$ probability the portfolio will lose an amount that is larger than the initial surplus within the next $n$ periods of time. In this case, the default will occur within the next $n$ periods of time and the process will stop before time $n$. The VaR can be interpreted as the maximum affordable loss that the company is willing to face. If equation 6 does not have a solution but equation 5 does, the VaR is less than the initial surplus.

2. In dealing with market risk, as investments become more liquid, VaR usually has a smaller time horizon (say one to ten days). Since credit is generally viewed over a longer time horizon, the time horizon for credit risk should be longer. A one-year time horizon is commonly used. However, recent developments and practical experience suggest that risk managers should pay attention to the impact of relatively short-term, market-driven factors on credit risk. On the other hand, our model can be used to set the margin requirements in order to ensure that the company will not face unaffordable loss with a given probability. This is different from using VaR to manage daily or weekly earnings from a mark to market perspective. In this case, we should use a longer time horizon VaR than usual (for example, one year or even longer).

We will now consider the case of infinity time horizon. The infinity time horizon model has its own merits in the context of integrated risk management. The importance given to it is justified by the different interpretations of the model, as given in the remarks below. Similar to the $n$-periods case, we can determine the VaR using the following equations:

For $y<u$

$$
P\left\{\Delta Y_{j} \leq-y, \exists j, T=\infty \mid I_{0}=i_{0}, U_{0}=u\right\}=5 \%,
$$

and for $y \geq u$

$$
P\left\{\Delta Y_{T} \leq-y, T<\infty \mid I_{0}=i_{0}, U_{0}=u\right\}=5 \% .
$$


The probability on the left hand side of (9) can be calculated from the following integral equations:

$$
\begin{gathered}
P\left\{\Delta Y_{j} \leq-y, \exists j, T=\infty \mid I_{0}=i_{0}, U_{0}=u\right\} \\
=\sum_{j=1}^{\infty} h_{j}^{i_{0}}(u, y) .
\end{gathered}
$$

Here,

$$
\begin{gathered}
h_{j}^{i_{0}}(u, y)=P\left\{\Delta X_{1}^{i_{0}}>-y, \ldots,-u<\Delta X_{1}^{i_{0}}+\cdots+\Delta X_{j}^{I_{j-1}} \leq-y, \ldots,\right. \\
\left.\Delta X_{1}^{i_{0}}+\cdots+\Delta X_{n}^{I_{n-1}}>-u, \ldots\right\}
\end{gathered}
$$

and $h_{j}^{i_{0}}(u, y)$ can be calculated as follows:

For $j=0$

$$
\begin{gathered}
h_{0}^{i_{0}}(-\infty)=0 \\
h_{0}^{i_{0}}(y)=\sum_{i=1}^{k} q_{i_{0} i} \int_{-y}^{\infty} h_{0}^{i}(y+x) d F^{i_{0}}(x) .
\end{gathered}
$$

For $j=1$

$$
h_{1}^{i_{0}}(u, y)=\sum_{i=1}^{k} q_{i_{0} i} \int_{-u}^{-y} h_{0}^{i}(u+x) d F^{i_{0}}(x)
$$

After obtaining $h_{1}^{i_{0}}(u, y)$, we consider the case $j \geq 2$ :

$$
h_{j}^{i_{0}}(u, y)=\sum_{i=1}^{k} q_{i_{0} i} \int_{-y}^{\infty} h_{j-1}^{i}(u+x, y+x) d F^{i_{0}}(x) .
$$

The probability on the left-hand side of (10) can be calculated using the following coupled Volterra-type integral equation system.

First, let

$$
H^{i_{0}}(u, y)=P\left\{\Delta Y_{T} \leq-y, T<\infty \mid I_{0}=i_{0}, U_{0}=u\right\} .
$$


be the distribution of the portfolio change, where $y \geq u$ Then

$$
\begin{gathered}
H^{i_{0}}(u, y)=P\left\{\Delta Y_{T} \leq-y, T<\infty \mid I_{0}=i_{0}, U_{0}=u\right\} \\
=\sum_{n=1}^{\infty} P\left\{\Delta Y_{t} \leq-y, T=n \mid I_{0}=i_{0}, U_{0}=u\right\} \\
=\sum_{n=1}^{\infty} P\left\{\Delta X_{1}^{i_{0}}>-u, \Delta X_{1}^{i_{0}}+\Delta X_{2}^{I_{1}}>-u, \cdots, \Delta X_{1}^{i_{0}}+\cdots\right. \\
\left.+\Delta X_{n-1}^{I_{n-2}}>-u, \Delta X_{1}^{i_{0}}+\cdots+\Delta X_{n}^{I_{n-1}} \leq-y\right\} \\
=\sum_{n=1}^{\infty} h_{n}^{i_{0}}(u, y) .
\end{gathered}
$$

Therefore, the distribution of the portfolio change satisfies the following coupled Volterra-type integral equation system:

$$
\begin{gathered}
H^{i_{0}}(u, y)=F^{i_{0}}(-y)+\sum_{i=1}^{k} q_{i_{0}} \int_{-u-}^{\infty} H^{i}(u+x, y+x) d F^{i_{0}}(x) \\
i_{0}=1,2, \ldots, k
\end{gathered}
$$

This system contains $k$ unknown functions $H^{1}(u, y), \ldots, H^{k}(u, y)$ and $k$ equations. The convergence of the above infinity summations and integrations is obvious.

\section{Remarks:}

1. The main issue that arises when using $\mathrm{VaR}$ as a risk management tool concerns how to calculate (or estimate) the portfolio's VaR. In the case of a change in the portfolio value following a normal distribution, this is a simple matter. However, as many researchers have noticed (see for example Duffie and Pan (1997) and Hull and White (1998)), the portfolio change distributions are fat-tailed. In Venkataraman (1997), a mixture of normal distributions was used to 
model the portfolio change. It was demonstrated that the mixture model provides a heavier tail distribution than normal. If we interpret our model differently, by regarding the Markov chain as a random process that is used to create a mixture random variable, we can also claim that we are using the Markov chain to create a heavier tail random variable. In this case, our model becomes a VaR model that uses a random mixture model to represent the portfolio change.

2. In actuarial science literature, there is a lot of work about distributional results on the severity of ruin (see, for instance, Gerber, Goovaerts and Kaas (1987) and Gerber and Shiu (1997)). The idea underlying the present paper stems from these discussions of the severity of ruin.

\section{A numerical example}

In this subsection, we use an example to illustrate the method employed in this paper. This example will be revisited in the next section. We use the one-year credit rating transition matrix from "Introduction to CreditMetrics." However, we use the conditional probabilities on the non-default states rather than the matrix directly.

$$
Q=\left[\begin{array}{ccccccc}
.9081 & .0833 & .0068 & .0006 & .0012 & 0 & 0 \\
.0070 & .9065 & .0779 & .0064 & .0006 & .0014 & .0002 \\
.0009 & .0227 & .9111 & .0552 & .0074 & .0026 & .0001 \\
.0002 & .0033 & .0596 & .8709 & .0531 & .0117 & .0012 \\
.0003 & .0014 & .0068 & .0781 & .8140 & .0893 & .0101 \\
0 & .0012 & .0025 & .0045 & .0684 & .8805 & .0429 \\
.0027 & 0 & .0028 & .0162 & .0296 & .1401 & .8086
\end{array}\right]
$$

We assume that the portfolio changes in each time interval are normally distributed. That is:

$$
\Delta X^{i} \sim N\left(\mu_{i}, \sigma_{i}^{2}\right)
$$

where $\sigma_{1}^{2}=1 / 4, \sigma_{2}^{2}=1, \sigma_{3}^{2}=4, \sigma_{4}^{2}=9 \quad \sigma_{5}^{2}=16, \sigma_{6}^{2}=25$ and $\sigma_{7}^{2}=36$ In figures 1 and 2 , we use $\mu_{1}=5, \mu_{2}=3, \mu_{3}=2, \mu_{4}=1$, 


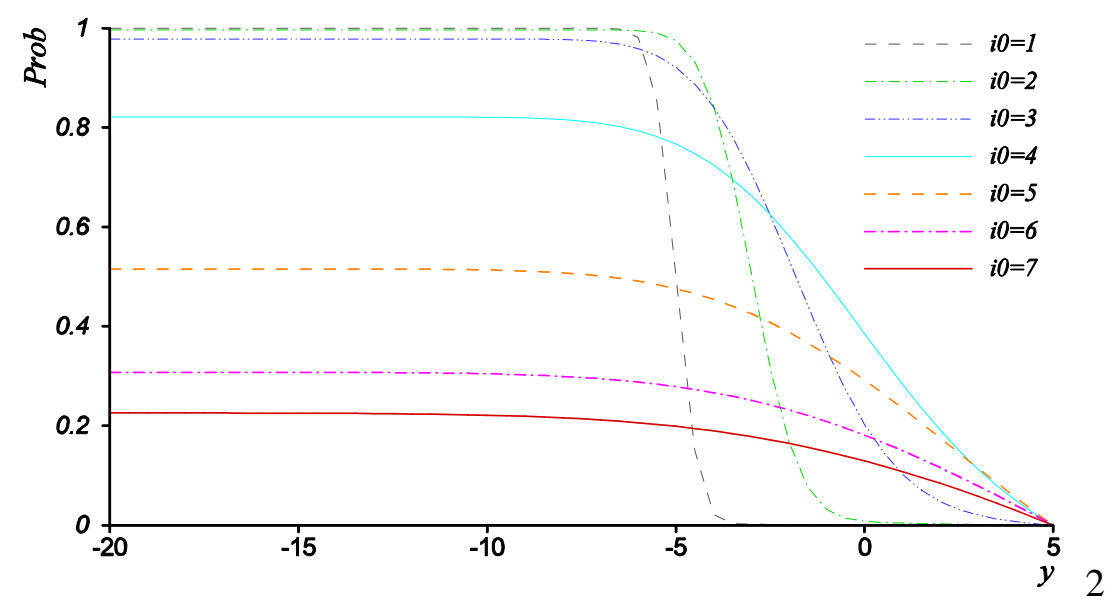

FIGURE 1.—VaR vs. confidence level $(y<u)$.

$\mu_{5}=0, \mu_{6}=-1$, and $\mu_{7}=-2$ and in figures 3 and 4 , we use $\mu_{1}=20, \mu_{2}$ $=15, \mu_{3}=10, \mu_{4}=5, \mu_{5}=3, \mu_{6}=0$, and $\mu_{7}=-2$. All the numerical results seem to be plausible.

Figure 1 gives the relationship between the probabilities on the lefthand side of equation 5 and the values of $y$ for $u=5$ and $n=8$. Here, we fix $u=5$. The initial surplus should affect the rating at time 0 (the same remark applies to all the examples below). Note that this figure does not indicate a clear, regular relation among the different ratings when $y \geq 0$. This is because when the rating is high, the probability of a portfolio loss in excess of $y$ will be low, and when the rating is low, the probability of a portfolio loss in excess of $y$ will be high but the probability of a portfolio loss being less than $u$ will be low. So the overall effect of the rating on the probabilities on the left-hand side of equation 5 is rather complex. When $-y$ becomes larger, the probabilities on the lefthand side of equation 5 approach the non-default probabilities.

Figure 2 gives the relationship between the probabilities on the lefthand side of equation 6 and the values of $y$ for $u=5$. It is clear that, if the rating is lower, the default probability will be higher. When the initial rating equals 5,6 , or 7 , equation 6 has a solution. For initial ratings equaling 1 to 4 , the $\mathrm{VaR}$ can be found from figure 1 .

Figures 3 and 4 give the solutions of the Volterra integral equation system 12 for $H$ as a function of $u$ when $y=2$ and $y=10$, respectively. 


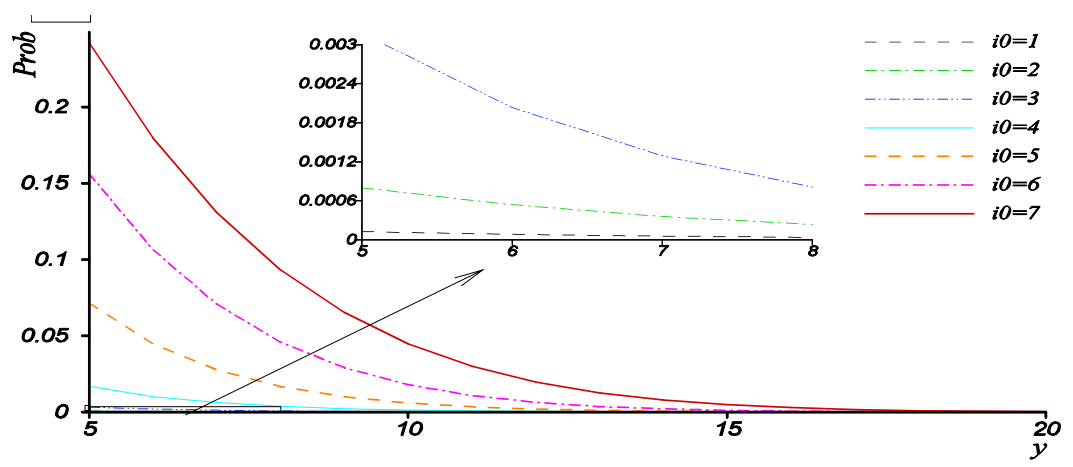

FigURE 2.- VaR vs. confidence level $(y>u)$.

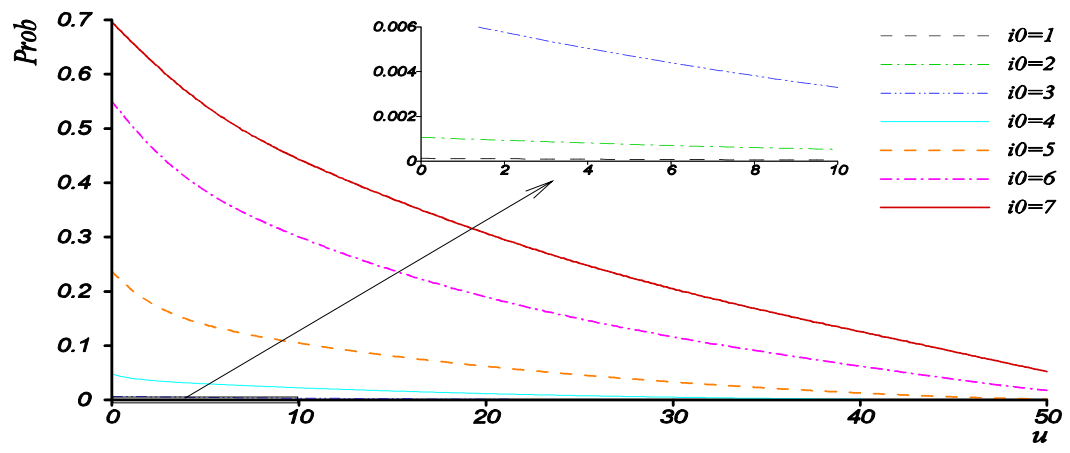

FIGURE 3.-Solution of (12) when $y=2$.

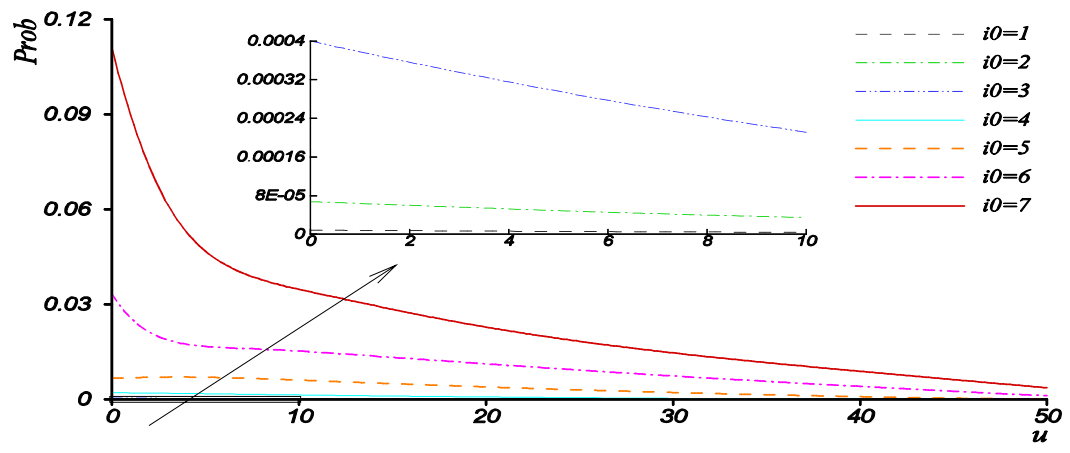

FIGURE 4.- Solution of (12) when $y=10$. 
These figures indicate the effect of the initial surplus on the VaR and default probabilities.

\section{Default Time and Default Probabilities}

As mentioned in the introduction, default has always been the main concern of most banks. The standard method of estimating default probabilities involves using historical data. This was the case, for example, with the JLT model or J. P. Morgan's CreditMetrics. In this section, we use the model and some of the results in Yang (2000) to discuss the default risk and default time.

\section{A. Default probability}

In the literature, the state space of the Markov chain includes the default state (see JLT (1997)). In practice, default is the state of most concern. We shall study the default risk using the estimated transaction probability matrix for credit ratings, but without including the default state. The credit rating model here is the same as in Section II.

The probability of default before or at time $n$ is defined as

$$
\psi_{n}^{i_{0}}(u)=P\left\{T \leq n \mid I_{0}=i_{0}, U_{0}=u\right\}
$$

and the ultimate default probability as

$$
\psi_{n}^{i_{0}}(u)=P\left\{T<\infty \mid I_{0}=i_{0}, U_{0}=u\right\}
$$

Let

$$
\varphi_{n}^{i_{0}}(u)=1-\psi_{n}^{i_{0}}(u)
$$

be the non-default probability. From Yang (2000), we have the following result:

Lemma 1. $\varphi_{n}^{i_{0}}(u)$ satisfies the following recursive equations: 


$$
\begin{gathered}
\varphi_{1}^{i_{0}}(u)=\bar{F}^{i_{0}}(-u)=1-F^{i_{0}}(-u), \\
\varphi_{n}^{i_{0}}(u)=\sum_{i=1}^{k} q_{i_{0} i} \int_{-u-}^{\infty} \varphi_{n-1}^{i}(u+y) d F^{i_{0}}(y),
\end{gathered}
$$

$n=2,3, \ldots$, and $\psi^{i_{0}}(u), i_{0}=1,2, \ldots, k$ satisfy the following coupled Volterra-type integral equation system:

$$
\psi^{i_{0}}(u)=F^{i_{0}}(-u)+\sum_{i=1}^{k} q_{i_{0} i} \int_{-u-}^{\infty} \psi^{i}(u+y) d F^{i_{0}}(y) .
$$

The proof of Lemma 1 is based on the recursive calculation that was proposed in De Vylder and Goovaerts (1988).

Example. We consider the example in Section II again. In table 1, we use $\mu_{1}=5, \mu_{2}=3, \mu_{3}=2, \mu_{4}=1, \mu_{5}=0, \mu_{6}=-1$, and $\mu_{7}=-2$. Table 1 gives the non-default probabilities calculated from the recursive formulas 14 and 15 for $u=5$.

Figure 5 gives the solution of the integral equation system 16 . The values of the parameters used here are the same as in figures 3 and 4 .

\section{B. Default time}

We now discuss the default time distribution. Let

$$
G_{n}^{i_{0}}(u)=P\left\{T=n \mid I_{0}=i_{0}, U_{0}=u\right\}
$$

Using the recursive method, we can obtain the default time distribution as follows:

Lemma 2. The distribution of the default time can be calculated using the following recursive equations:

$$
G_{1}^{i_{0}}(u)=F^{i_{0}}(-u)
$$


TABLE 1. Non-default Probabilities

A. $1 \leq n \leq 4$

$\begin{array}{llccc}n & 1 & 2 & 3 & 4 \\ i_{0}=1 & 1.00000 & 0.999993 & 0.999952 & 0.999882 \\ i_{0}=2 & 1.00000 & 0.999836 & 0.999475 & 0.998976 \\ i_{0}=3 & 0.999775 & 0.998005 & 0.995219 & 0.992010 \\ i_{0}=4 & 0.977373 & 0.941426 & 0.911119 & 0.886701 \\ i_{0}=5 & 0.894496 & 0.784091 & 0.704873 & 0.646862 \\ i_{0}=6 & 0.788239 & 0.624959 & 0.521345 & 0.450873 \\ i_{0}=7 & 0.691513 & 0.509285 & 0.407070 & 0.342946\end{array}$

B. $5 \leq n \leq 8$

\begin{tabular}{lcccc}
$n$ & 5 & 6 & 7 & 8 \\
$i_{0}=1$ & 0.999789 & 0.999677 & 0.999548 & 0.999400 \\
$i_{0}=2$ & 0.998377 & 0.997703 & 0.996965 & 0.996171 \\
$i_{0}=3$ & 0.988622 & 0.985161 & 0.981674 & 0.978181 \\
$i_{0}=4$ & 0.866576 & 0.849505 & 0.834661 & 0.821490 \\
$i_{0}=5$ & 0.602594 & 0.567606 & 0.539171 & 0.515533 \\
$i_{0}=6$ & 0.399993 & 0.361595 & 0.331627 & 0.307606 \\
$i_{0}=7$ & 0.299330 & 0.267885 & 0.244199 & 0.225712 \\
\hline
\end{tabular}

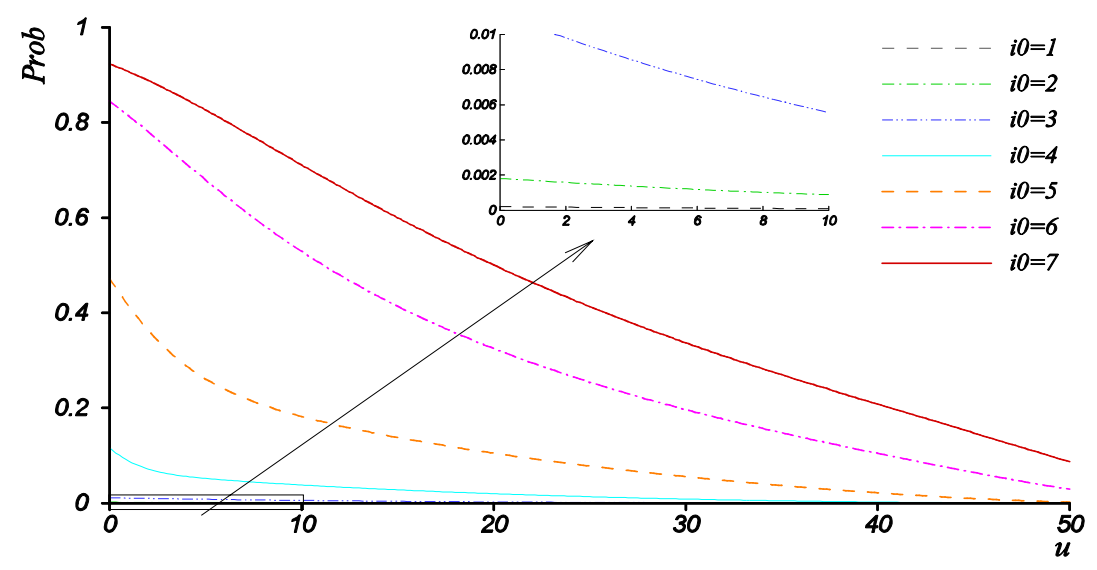

FIGURE 5.-Solution of (16) 
TABLE 2. Default Time Distribution

$\begin{array}{lcccc}\text { A. } 1 \leq n \leq 4 & & & \\ n & 1 & 2 & 3 & 4 \\ i_{0}=1 & 0.486887 \mathrm{E}-197 & 0.117773 \mathrm{E}-06 & 0.482978 \mathrm{E}-05 & 0.116431 \mathrm{E}-04 \\ i_{0}=2 & 0.619335 \mathrm{E}-38 & 0.204812 \mathrm{E}-04 & 0.668437 \mathrm{E}-04 & 0.985179 \mathrm{E}-04 \\ i_{0}=3 & 0.990179 \mathrm{E}-09 & 0.934214 \mathrm{E}-04 & 0.276415 \mathrm{E}-03 & 0.408816 \mathrm{E}-03 \\ i_{0}=4 & 0.123062 \mathrm{E}-03 & 0.184695 \mathrm{E}-02 & 0.250688 \mathrm{E}-02 & 0.262296 \mathrm{E}-02 \\ i_{0}=5 & 0.621508 \mathrm{E}-02 & 0.160854 \mathrm{E}-01 & 0.132180 \mathrm{E}-01 & 0.103645 \mathrm{E}-01 \\ i_{0}=6 & 0.359499 \mathrm{E}-01 & 0.416151 \mathrm{E}-01 & 0.264394 \mathrm{E}-01 & 0.176693 \mathrm{E}-01 \\ i_{0}=7 & 0.912453 \mathrm{E}-01 & 0.643123 \mathrm{E}-01 & 0.338848 \mathrm{E}-01 & 0.199415 \mathrm{E}-01\end{array}$

B. $5 \leq n \leq 8$

$\begin{array}{lcccc}n & 5 & 6 & 7 & 8 \\ i_{0}=1 & 0.177801 \mathrm{E}-04 & 0.235274 \mathrm{E}-04 & 0.289968 \mathrm{E}-04 & 0.342840 \mathrm{E}-04 \\ i_{0}=2 & 0.122581 \mathrm{E}-03 & 0.143453 \mathrm{E}-03 & 0.162791 \mathrm{E}-03 & 0.181181 \mathrm{E}-03 \\ i_{0}=3 & 0.504224 \mathrm{E}-03 & 0.576666 \mathrm{E}-03 & 0.633956 \mathrm{E}-03 & 0.680414 \mathrm{E}-03 \\ i_{0}=4 & 0.258784 \mathrm{E}-02 & 0.250603 \mathrm{E}-02 & 0.240961 \mathrm{E}-02 & 0.230989 \mathrm{E}-02 \\ i_{0}=5 & 0.829401 \mathrm{E}-02 & 0.679455 \mathrm{E}-02 & 0.567713 \mathrm{E}-02 & 0.482015 \mathrm{E}-02 \\ i_{0}=6 & 0.125328 \mathrm{E}-01 & 0.930653 \mathrm{E}-02 & 0.715621 \mathrm{E}-02 & 0.565477 \mathrm{E}-02 \\ i_{0}=7 & 0.128193 \mathrm{E}-01 & 0.879717 \mathrm{E}-02 & 0.634642 \mathrm{E}-02 & 0.476295 \mathrm{E}-02\end{array}$

$$
G_{n}^{i_{0}}(u)=\sum_{i=1}^{k} q_{i_{0} i} \int_{-u-}^{\infty} G_{n-1}^{i}(u+y) d F^{i_{0}}(y)
$$

The notion of default time distribution is useful in credit derivatives. Suppose a defaultable contingent claim pays $\varsigma>0$ (random) at time $S$ if the default does not occur before time $S$. If the default occurs before time $S$, the payment will be either 0 or a reduced amount. The calculation of the price of this contingent claim involves the distribution of the default time. Hence, the calculation of a default time distribution is part of the calculation of the default derivative price.

Example. We continue the example in Section II. The following table (table 2) provide some values of the default time distribution when the initial surplus $u=5$ and the rest of the parameters are the same as in table 1. 


\section{Concluding Remarks}

This paper has proposed an integrated risk management method. The model described here can capture the market risk, credit rating change, and default risk. Using some recursive equations and coupled Volterra integral equation systems, a procedure for calculating the VaR of the portfolio has been provided. However, this paper has only focused on ideas and theoretical insight and has not sought to apply the method to practical problems.

Limitations are a fact of life. It is very difficult, maybe even impossible, to find a perfect risk management methodology. We are not claiming that the method proposed in this paper is superior to those in the literature. Indeed, there are some disadvantages to this model. The model, as it stands, is rather simple. We assume that the rating state depends on the initial surplus, but we cannot assume, for mathematical reasons, that the Markov chain will depend on the surplus process. The numerical calculations are complex and computationally intensive. With today's high level of computing power, hopefully this problem is not so serious. There are many problems that need to be considered further. For general distributions, the stability of numerical solutions of the coupled Volterra integral equation system needs to be investigated carefully. Up to now, we have only considered the discrete time model. The ideas in this paper can be used for other risk models as well. We shall address these problems in future research.

\section{References}

Arvanitis, A.; Gregory, J.; and Laurent, J. P. 1999. Building models for credit spreads. The Journal of Derivatives 6: 27-43.

De Vyldrer, F., and Goovaerts, M. J. 1988. Recursive calculation of finite-time ruin probabilities. Insurance: Mathematics and Economics 7:1-7.

Duffie, D., and Pan, J. 1997. An overview of value at risk. Journal of Derivatives Spring, 7-49.

Embrechts, P. 2000. Value at Risk. Lecture Notes for the Workshop in the Center of Financial Time Series. The University of Hong Kong.

Gerber, H. U.; Goovaerts, M. J.; and Kaas, R. 1987. On the probability and severity of ruin. ASTIN Bulletin 17: 151-163.

Gerber, H. U., and Shiu, E. S. W. 1997. The joint distribution of the time of ruin, the surplus immediately before ruin and the deficit at ruin. Insurance: 
Mathematics and Economics 21: 129-137.

Huang, H., 2000. Tie the knot. Risk (March): 20-22.

Hull, J. C., and White, A. 1998. Value at risk when daily changes in market variables are not normally distributed. Journal of Derivatives 5: 9-19.

Jarrow, R. A.; Lando, D.; and Turnbull, S. M. 1997. A Markov model for the term structure of credit risk spread. Review of Financial Studies 10: 481523.

Kijima, M., and Komoribayashi, K. 1998. A Markov chain model for valuing credit risk derivatives. The Journal of Derivatives 5: 97-108.

Morgan, J. P. 1996. RiskMetrics ${ }^{\mathrm{TM}}$ - Technical Document. New York.

Morgan, J. P. 1997. Introduction to CreditMetrics. New York.

Ong, M. K. 1999. Internal Credit Risk Models. Risk Books, London.

Steinherr, A. 1988. Derivatives: The Wild Beast of Finance. Wiley, New York.

Venkataraman, S. 1997. Value at risk for a mixture of normal distributions: The use of quasi-Bayesian estimation techniques. Economic Perspectives: 3-13.

Yang, H. 1999. Non-exponential bounds for ruin probability with interest effect included. Scandinavian Actuarial Journal: 66-79.

Yang, H. 2000. Ruin theory in a financial corporation model with credit risk. Submitted. 